\title{
Evaluer et communiquer les incertitudes associées aux prévisions hydrologiques pour mieux partager l'information
}

Lionel BERTHET ${ }^{a}$, Éric GAUME, Olivier PIOTTE ${ }^{c}$ et les groupes de travail du Conseil scientifique et technique du SCHAPI ${ }^{1}$ et du chantier d'intérêt commun SCHAPI - SPC Estimation des incertitudes de prévision ${ }^{2}$

\footnotetext{
a. Service de prévision des crues (SPC) Loire - Cher - Indre (direction régionale de l'Environnement de l'Aménagement et du Logement du Centre-Val de Loire), lionel.berthet@developpement-durable.gouv.fr

b. IFSTTAR, département Géotechnique, Environnement, Risques naturels et Sciences de la Terre (Bouguenais), eric.gaume@ifsttar.fr

c. Service central d'Hydrométéorologie et d'Appui à la Prévision des Inondations (MEDDE / DGPR / SRNH / SCHAPI),

olivier.piotte@developpement-durable.gouv.fr
}

RÉSUMÉ. - Dans le cadre de la modernisation du service Vigicrues (www.vigicrues.gouv.fr) et plus généralement de l'amélioration du dispositif de prévision des crues en France, le $\mathrm{SCHAPI}^{3}$ a engagé en 2012 une réflexion sur l'estimation et la communication des incertitudes associées aux prévisions hydrologiques. Cette réflexion a été confiée à deux groupes de travail. Le premier, composé de membres du Conseil scientifique et technique du SCHAPI a mené une réflexion sur les conditions et les modalités de production de prévisions, articulée autour de trois questions : le degré d'incertitude acceptable pour les utilisateurs, l'estimation quantitative des incertitudes, la représentation et la communication des incertitudes. Le second groupe, constitué d'agents du SCHAPI et de services de prévision des crues a été chargé de proposer et de tester des solutions techniques pour l'estimation et la publication des incertitudes associées aux prévisions. Les principales conclusions de ces deux groupes permettent d'apprécier ce que pourrait être l'évolution des produits de prévision des crues et des inondations à court terme. Les principaux obstacles à la diffusion des incertitudes de prévision ne sont cependant pas techniques, mais institutionnels et sociologiques. Un important travail de préparation des principaux utilisateurs des prévisions hydrologiques, notamment les acteurs de la gestion de crise, sera donc nécessaire avant que la diffusion de ces prévisions enrichies puisse être pleinement efficace.

Mots-clés : prévision des crues, incertitude, gestion de crise

\section{Estimation and communication of uncertainties related to hydrological forecasts for a better information sharing}

\begin{abstract}
To prepare the modernization of the flood vigilance system (www.vigicrues.gouv.fr) and more generally to improve the French flood forecasting system, the $\mathrm{SCHAPI}^{3}$ has launched in 2012 a reflection on the estimation and communication of uncertainties related to hydrological forecasts. Two think tanks were created for this purpose. The first, composed of members of the SCHAPI's scientific board had to answer to three questions, namely: what uncertainty level is acceptable for the end-users, how should uncertainties be estimated and how should they be represented to be communicated. The second think tank, composed of engineers and forecasters from the SCHAPI and flood forecasting services, had to suggest and test technical solutions for the evaluation and the publication of forecasting uncertainties. The main outcomes of these two think tanks are presented hereafter. They give a glimpse of the possible evolutions of flood forecasting products in the near future. It is important to note, that the main obstacles to the communication of forecasting uncertainties are not technical but rather institutional and sociological. The expectations as well as the uses of the forecasts will have to be rethought in depth to account for the new kind of information represented by uncertainties. Therefore, in parallel with the technological developments, an important preparation work, conducted with the end-users, will be necessary before forecasting uncertainties can efficiently be communicated.
\end{abstract}

Key-words: flood forecasting, uncertainty, crisis management

1. Bruno Janet (SCHAPI), Bernard Chastan (IRSTEA), Denis Dartus (IMFT), Guy Delrieu (CNRS, UMR 5564), Patrick Fourmigué (Cerema), Rémy Garçon (EDF-DTG), Jean-Michel Grésillon (IRSTEA), Daniel Jouve (CNR), Murielle Lafaye (CNES), Céline Lutoff (CNRS UMR 5564), Éric Martin (Météo France), Charles Obled (CNRS, UMR 5564), Jutta Thielen (Commission européenne, JRC-Ispra)

2. Constantin Ardilouze, Lionel Berthet, Olivier Boujard, Christian Brousse, Carine Chaléon, Marie-Pierre Cribellier, Alain Gautheron, Johan Habert, Antoine Herman, Bruno Janet, Nicolas Jurdy, Éric Kermarec, Éric Ladegaillerie, Renaud Marty, Jean Pansu, Olivier Piotte, Didier Reinbold, Matthieu Saunier, Jean-Pedro Silva et Pascal Villenave

3. Service central d'Hydrométéorologie et d'Appui à la Prévision des Inondations 


\section{I. ÉLÉMENTS DE CONTEXTE}

Les prévisions, et notamment les prévisions hydrométéorologiques, sont par nature incertaines. Comme le disait l'humoriste danois R. Storm Petersen, qu'aimait citer le physicien Niels Bohr : " il est difficile de faire des prédictions, surtout sur l'avenir ». Le degré d'incertitude associé aux prévisions est de plus un élément d'information essentiel lorsqu'il s'agit de prendre une décision. Or, ce degré d'incertitude est rarement évalué et diffusé, pour des raisons techniques - manque d'outils pour l'estimation des incertitudes, mais aussi et surtout pour des raisons liées aux pratiques actuelles des services de prévision et aux attentes de leurs utilisateurs. Du point de vue des utilisateurs, l'affichage des incertitudes peut être perçu comme un frein à la prise de décisions et une forme de transfert de responsabilité du prévisionniste vers l'usager. Les prévisionnistes, de leur côté sont souvent réticents à l'idée de dévoiler des niveaux d'incertitude élevés qui pourraient mettre en cause leurs compétences et expertises ${ }^{4}$.

Les exemples de diffusion d'incertitudes associées aux prévisions hydrométéorologiques sont donc restés longtemps peu nombreux, essentiellement cantonnés au champ des prévisions météorologiques de moyenne échéance (prévisions d'ensemble, indices de confiance). À ces échéances, les actions engagées sur la base des prévisions sont limitées et relativement peu « coûteuses » (vigilance, activation d'astreintes), facilitant l'acceptation des incertitudes. Lorsque les décisions d'actions ont des conséquences bien plus importantes (interruption d'activités et de trafic, évacuation), l'aversion à l'incertitude des décideurs publics ou privés augmente. Elle correspond à une répugnance à la prise de risque qui a longtemps limité la réflexion sur l'incertitude et les possibilités d'évolution des dispositifs de prévision existants.

Les réflexions conduites dans de nombreux pays sur le sujet [Berthet et Piotte, 2014] et les réactions à la suite d'événements récents, identifiant le manque d'information sur les incertitudes de prévision comme la cause de décisions inadaptées ${ }^{5}$, indiquent cependant que les choses évoluent actuellement. C'est dans ce contexte et en vue d'améliorer le dispositif actuel de prévision des crues et des inondations que le SCHAPI a engagé une réflexion sur l'évaluation et la communication des incertitudes associées aux prévisions hydrologiques, en confiant cette réflexion à deux groupes de travail :

- le premier, composé de membres du Conseil scientifique et technique (CST) du SCHAPI, a construit sa réflexion autour de 3 questions : le degré d'incertitude acceptable pour les utilisateurs, l'estimation quantitative des incertitudes, la représentation et la communication des incertitudes [SCHAPI, 2013],

- le second, composé de prévisionnistes du SCHAPI et des SPC, a travaillé sur l'estimation quantitative des incertitudes et la publication opérationnelle des prévisions [SCHAPI SPC, 2014].

4. Des expériences révèlent que les prévisionnistes ont tendance naturellement à sous-évaluer les incertitudes associées à leurs prévisions. Ce biais disparait lorsqu'il leur est demandé d'effectuer des paris sur la base de leurs prévisions, signe de leur conscience des niveaux d'incertitude, mais des réticences à les expliciter [Houdant, 2004].

5. Les mesures drastiques prises à New York le 27 janvier 2015, en réponse aux prévisions pessimistes des effets de la tempête Juno, ont par exemple créé la polémique aux États-Unis et conduit le directeur du National Weather Service à déclarer : "Nous devons énoncer ces incertitudes clairement de manière à ce que les responsables puissent évaluer le risque ».
Le texte qui suit reprend les principales conclusions de ces deux groupes de travail complémentaires, qui préfigurent les évolutions du dispositif français de prévision des crues et des inondations.

\section{RÉFLEXIONS ET RECOMMANDATIONS DU CONSEIL SCIENTIFIQUE (CST) DU SCHAPI}

Le groupe de travail s'est fondé sur les exemples étrangers et s'est aussi beaucoup inspiré des réflexions approfondies menées à Électricité de France sur le sujet [Houdant, 2004]. Il a proposé des recommandations méthodologiques et techniques concernant l'estimation des incertitudes, leur mode de représentation ou encore l'évaluation des prévisions « incertaines ». Mais ses conclusions les plus importantes sont d'un autre ordre. Les principaux obstacles à la communication des incertitudes de prévision sont en effet institutionnels et sociologiques : rien ne sera réellement possible sans une évolution du regard et des attentes par rapport aux prévisions et à la gestion des crises.

La première question posée au groupe illustre bien les contraintes actuelles pesant sur les prévisions et qui limitent les possibilités d'évolution : " quel est le degré d'incertitude acceptable pour les divers utilisateurs des prévisions ?». Cette formulation intègre la prévision dans la prise de décision et laisse entendre que le niveau d'incertitude pourrait être choisi.

Or, le niveau d'incertitude n'est pas choisi. On peut espérer qu'il diminue avec le temps et l'amélioration des connaissances scientifiques et des dispositifs d'observation, mais c'est une donnée du problème. De plus, la prévision est une information importante certes, mais une information parmi d'autres sur laquelle est assise la prise de décision. La véritable question que le décideur doit se donc poser est plutôt : « comment organiser la prise de décision et quelles actions sont possibles compte tenu du niveau d'incertitude? »

Le groupe de travail a donc consacré un temps important à l'élaboration d'éléments de diagnostic sur lesquels il faut arriver à s'entendre avec les utilisateurs des prévisions pour préparer le terrain à la communication des incertitudes avant de proposer des recommandations.

\section{II.1. Éléments de diagnostic}

1. Une prévision hydrométéorologique est une information parmi d'autres, nécessairement incertaine, mise à disposition pour la prise de décision. La production de cette information et la décision doivent être dissociées. Le rôle du prévisionniste est de fournir une information expertisée, la plus exacte et précise possible compte tenu de l'état actuel des connaissances. Les incertitudes croissent lorsque l'horizon de prévision augmente. Elles sont aussi plus importantes pour les événements exceptionnels, sur lesquels l'expertise n'a eu que peu ou pas l'occasion de s'exercer. C'est justement pour ces événements que les prévisions hydrologiques sont essentiellement requises.

2. Les incertitudes associées aux prévisions font partie intégrante de l'information ${ }^{6}$. Cette information est indispensable pour une prise de décision éclairée (encadré 1).

6. Si les incertitudes ne leur sont pas fournies, les utilisateurs estiment des incertitudes de prévision sur la base de leur propre expérience pour prendre leurs décisions, comme l'a montré une enquête sur les prévisions météorologiques auprès de la population des États Unis [Morss et al., 2008]. 
Les services de prévision font au mieux en fonction de l'état actuel des connaissances (état de l'art) et de leurs moyens. Le niveau d'incertitude n'est pas choisi mais subi : c'est une donnée du problème.

Encadré 1: Si le seuil d'apparition des dommages dans une section de cours d'eau est de $130 \mathrm{~m}^{3} / \mathrm{s}$, dire que le débit prévu est de $110 \mathrm{~m}^{3} / \mathrm{s}$, ou bien qu'il y a $90 \%$ de chances pour que ce débit se situe entre 90 et $150 \mathrm{~m}^{3} / \mathrm{s}$, ou bien encore qu'il y a $30 \%$ de chances pour que le seuil de $130 \mathrm{~m}^{3} / \mathrm{s}$ soit dépassé, n'a pas le même contenu informatif. La première proposition, négligeant les incertitudes, peut être trompeuse.

3. L'incertitude n'empêche pas la décision. C'est au contraire l'un des critères de décision (encadré 2). L'incertitude et la prise de risque sont au cœur de toute décision. Décider, c'est faire un choix et souvent prendre un risque ${ }^{7}$. La non-décision n'existe pas : reculer la décision d'engager une action dans l'attente d'une information plus précise conduit à réduire le délai d'anticipation et à limiter les possibilités d'action, en échange d'un supplément d'information. Les utilisateurs doivent donc arbitrer entre niveau de précision et délai d'anticipation en fonction du type de décision, de l'enjeu, de leurs capacités d'action et du coût de ces actions (astreintes, déclenchement d'alertes, mobilisation de moyens, modification ou arrêt d'activités, interventions, évacuations...).

7. L'étude des comportements décisionnels des acteurs en situation de risque est l'un des sujets, si ce n'est le sujet central, des travaux de recherche en théorie de la décision (Savage, Von Neuman \& Morgenstern, Allais)
Encadré 2 : En cas de crue centennale, les caves d'un musée seront inondées. Pour évacuer l'ensemble des collections stockées dans ces caves, il faut au moins 3 jours. Or le taux de fausses alertes du dépassement du seuil d'inondation des caves du musée à 3 jours dépasse $90 \%$ (9 fausses alertes sur 10 alertes). Face à cet état de fait, le gestionnaire du musée peut prendre trois décisions dépendant de son évaluation de l'utilité des fonds et des coûts de mise en sécurité :

1. accepter d'évacuer l'ensemble des caves à chaque alerte (en moyenne une fois tous les dix ans) pour être à peu près certain de sauver les collections ;

2. déménager définitivement les collections, car le coût de ces déménagements répétés est trop important ;

3. renoncer à sauver l'ensemble des collections en cas de crue et soit envisager de ne rien déménager ou bien de déménager uniquement la partie la plus précieuse des collections sur la base de prévisions plus précises à échéance plus courte (24 heures par exemple).

4. La réduction des incertitudes associées aux valeurs prévues est loin d'être le seul moyen d'enrichir et de préciser les prévisions hydrologiques (figure 1). La multiplication des échéances de prévision, la proposition de faisceaux de trajectoires, le repérage de valeurs de cotes ou de débits de référence et l'association de cartes de zones inondées, de localisation des enjeux majeurs, le lien avec des actions prévues dans les plans communaux de sauvegarde sont autant d'informations, qui associées aux prévisions, peuvent largement amoindrir l'impact des incertitudes des prévisions pour la prise de décisions par les utilisateurs. Le débat sur les incertitudes ne doit pas être un facteur de blocage limitant les évolutions des services de prévision et des services de gestion de crise.

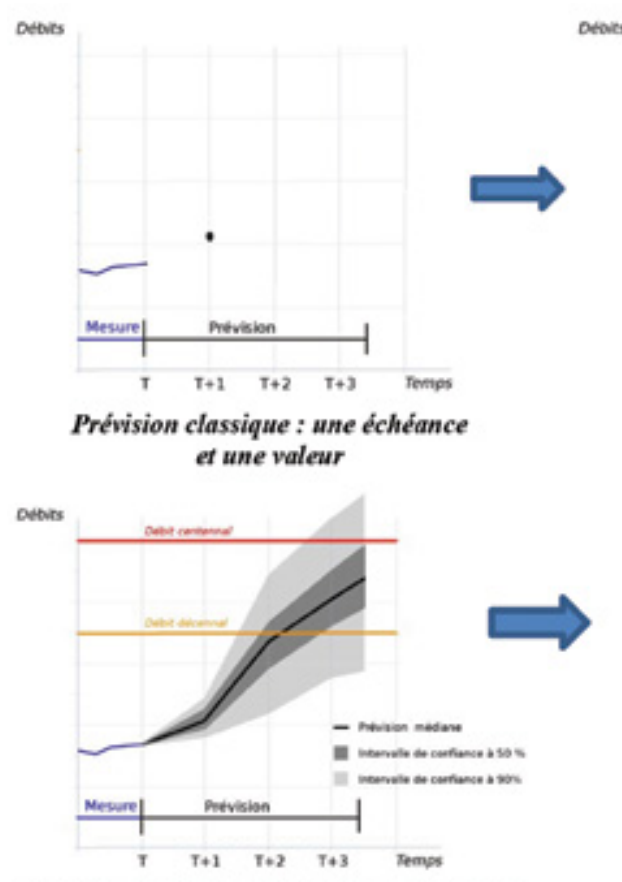

Positionnement par rapport à des références
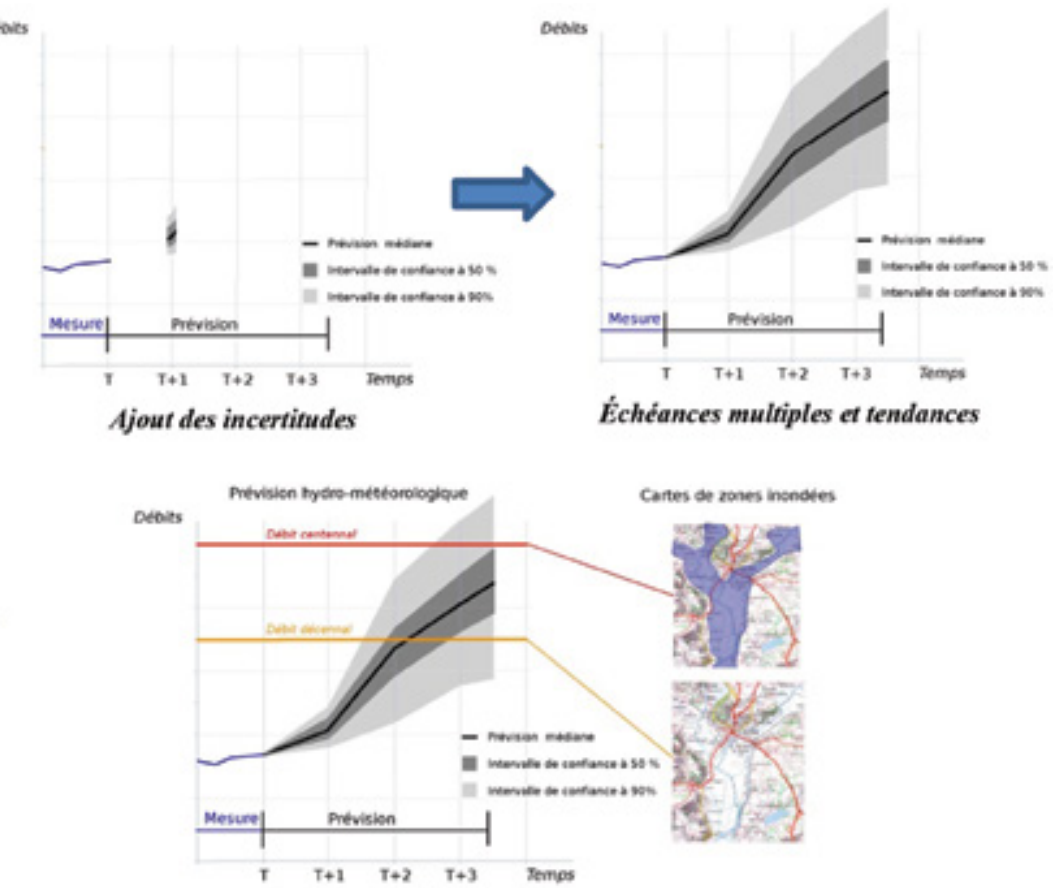

Ajout d'informations complémentaires : zones inondables, enjeux majeurs, routes inondées, actions du PCS

Figure 1 : Illustration de l'enrichissement possible des prévisions hydrologiques. 


\section{II.2. Recommandations}

1. Il est indispensable d'imposer l'idée que la prévision est une information expertisée parmi d'autres. La production de cette information et les prises de décisions doivent être clairement dissociées.

2. Les méthodes proposant une fourchette de valeurs prévues - hypothèses haute et basse ou intervalle de confiance de cote ou d'extension de la zone inondée à une échéance donnée - semblent a priori les plus simples à mettre en œuvre et les plus adaptées à la prévision des inondations (cf. exemple de la figure 1). C'est le choix arrêté dans de nombreux pays. Le développement des outils devra se faire en concertation avec les utilisateurs principaux que sont les services d'intervention et de secours. Une attention particulière devra être portée au traitement des échéances de prévision variables, nettement plus faibles ou plus importantes que les 24 heures de la mission réglementaire de vigilance.

3. L'information apportée sur l'incertitude des prévisions ne sera utile aux gestionnaires de crise, pour des prises de décisions en temps réel, que s'ils sont en capacité de les intégrer dans la prise de décision, c'est-à-dire s'ils disposent de règles et d'outils de prise de décision les prenant en compte. Ces outils doivent être construits en amont : détermination de seuils de déclenchement des actions par exemple. Dans le même ordre d'idée, la sensibilisation et la formation des principaux utilisateurs à l'exploitation des incertitudes de prévision devront être mis en place [Nobert et al., 2010].

4. Des outils d'estimation automatique des incertitudes sont indispensables dans la mesure où les prévisionnistes hydrologues changent fréquemment. De même, pour favoriser l'apprentissage et la construction d'expertise au sein des services de prévision, il est souhaitable que des prévisions soient produites tous les jours, même hors périodes de crues. Enfin, les prévisions devront être archivées, ainsi que les incertitudes estimées pour permettre l'évaluation des prévisions $^{8}$ et l'amélioration des méthodes. L'évaluation des modèles d'estimation des incertitudes nécessite de nombreux événements de crues. Elle est plus aisée pour des phénomènes courants, mais ne pourra reposer sur les données hydrométriques prises séparément sur chaque site pour des événements rares : les effectifs seront statistiquement insuffisants. Il sera donc nécessaire d'élaborer des méthodes d'évaluation globales des modèles de prévision à l'échelle de grandes régions.

5. Le temps jouera un rôle important pour la prise en main par les prévisionnistes et les utilisateurs de nouveaux outils de prévisions. Mais seule une mise en œuvre opérationnelle des outils permettra réellement leur amélioration et leur fiabilisation.

\section{III. ÉVOLUTION DES PRATIQUES AU SEIN DU RÉSEAU SCHAPI - SPC}

Fort des réflexions du groupe de travail du CST, le réseau du SCHAPI et des SPC se prépare à estimer les incertitudes associées à leurs prévisions, et à leur valorisation auprès de ses usagers. Ce travail engagé en 2012 a permis de fixer les grandes lignes opérationnelles qui sont présentées dans cette partie.

8. Des méthodes adaptées d'évaluation des prévisions avec incertitudes doivent être mises en œuvre, qui nécessitent des historiques de données importants. Il faut pouvoir vérifier en effet que le nombre observé de valeurs observées en dehors des fourchettes prévues est en accord avec la probabilité théorique associée à cette fourchette.

\section{III.1. L'objectif : représenter graphiquement les prévisions et les incertitudes associées sur Vigicrues}

À ce jour, des prévisions quantitatives de cote ou de débit sont publiées sous forme de tableaux (plus rarement, de graphes) dans des bulletins téléchargeables sur le site www.vigicrues.fr. Or le SCHAPI et les SPC disposent aujourd'hui d'outils et modèles de prévision leur permettant désormais de fournir des prévisions quantitatives pour de nombreux sites. Pour mieux valoriser cette information, le SCHAPI et les SPC se donnent pour objectif de fournir ces prévisions directement sur les graphes des pages des stations du site Vigicrues, en prolongement des observations qui y figurent actuellement.

La forme de ces graphes suit les recommandations du groupe de travail du CST. Elle est fixée pour apporter une information à la fois bien définie, accessible au grand public et utile à la gestion de crise : la prévision sera représentée par une tendance centrale encadrée de deux tendances correspondant à un intervalle de prévision. Celui-ci est défini par les quantiles $q_{0,1}$ et $q_{0,9}$. Ce choix présente plusieurs avantages. Tout d'abord, il assure l'homogénéité de l'information sur l'ensemble des points de prévision du territoire français, ce qui facilitera l'appropriation du message et la lecture des usagers intéressés par plusieurs sites. Par ailleurs, il permet d'offrir plusieurs niveaux de lecture, allant de la prise en compte d'une information qualitative ("les scénarios optimistes et pessimistes sont les niveaux qui ont peu de chance d'être dépassés ») à l'emploi de l'information quantitative autorisant la mise au point et l'utilisation de règles de gestion de crise précises valorisant cette information.

Pour atteindre cet objectif et le rendre pleinement utile, trois actions sont menées parallèlement : le développement des outils pratiques permettant aux SPC d'estimer l'incertitude de leurs prévisions (a minima, l'estimation de ces deux quantiles), l'accompagnement des prévisionnistes dans la prise en main de ces outils et surtout dans l'approfondissement de la culture des incertitudes, et enfin l'accompagnement des usagers de cette information pour en faciliter la bonne compréhension et, si besoin, pour aider à en faire le meilleur usage.

\section{III.2. Les outils en cours de développement au sein des services de l'État}

Le temps est une des denrées les plus précieuses en crise. Lors d'une crue, les services de prévision doivent être en mesure de produire des prévisions en temps limité, sur un nombre de sites potentiellement important (événements généralisés de crue). La plus-value des prévisionnistes (par rapport aux outils de prévision automatiques) résidant dans leur expertise, un cadre de travail facilitant au mieux l'expression de cette expertise est recherché à travers la mise en place de deux outils :

- un premier outil, développé par l'IRSTEA', fournissant une estimation "par défaut» de l'incertitude associée à une prévision déterministe calculée par un outil automatique de prévision (modèle...) ;

- un second outil, développé au sein du réseau SCHAPI SPC, permettant de modifier les prévisions automatiques en

9. Outil automatique d'estimation de l'incertitude prédictive en prévision hydrologique (OTAMIN) développé par l'unité de recherche Hydrosystèmes et Bioprocédés. 
quelques mouvements de souris et d'exprimer ainsi l'expertise humaine.

Le premier outil se fonde sur le travail mené par F. Bourgin et propose deux méthodes d'estimation de l'incertitude par analyse a posteriori des erreurs passées d'un outil de prévision déterministe : la régression des quantiles [Weerts et al., 2011] et QUOIQUE [Bourgin, 2014]. Cette approche compte parmi les méthodes les plus employées dans les services de prévision à travers le monde. Elle construit une statistique des erreurs passées en fonction de deux prédictants, la valeur de la variable prévue et l'horizon de prévision, à partir de séries d'erreurs déjà observées (en rejeu ou temps réel dans le passé). En temps réel, cette statistique est utilisée pour estimer les quantiles de la distribution de probabilité de la variable à prévoir (conditionnellement à la prévision déterministe et à l'horizon de prévision), ce qui donne une description succincte de l'incertitude de prévision (figure 2).

Cette méthode permet de ne faire que très peu d'hypothèses sur les distributions des grandeurs prévues (en dehors d'une hypothèse forte de stationnarité de la distribution de probabilité conditionnellement aux prédictants). Comme la plupart des approches disponibles, elle atteint ses limites sur les événements exceptionnels, pour lesquels nous disposons de peu voire aucune connaissance passée pour construire la statistique : une extrapolation, toujours périlleuse, doit être acceptée.

Le second outil est un « espace de travail» dans lequel le prévisionniste examine les prévisions (qui incluent les estimations des incertitudes associées) représentées graphiquement et les modifie s'il le juge nécessaire en fonction des sources d'incertitude qu'il identifie et de sa connaissance de l'écart entre le comportement du terrain (bassin

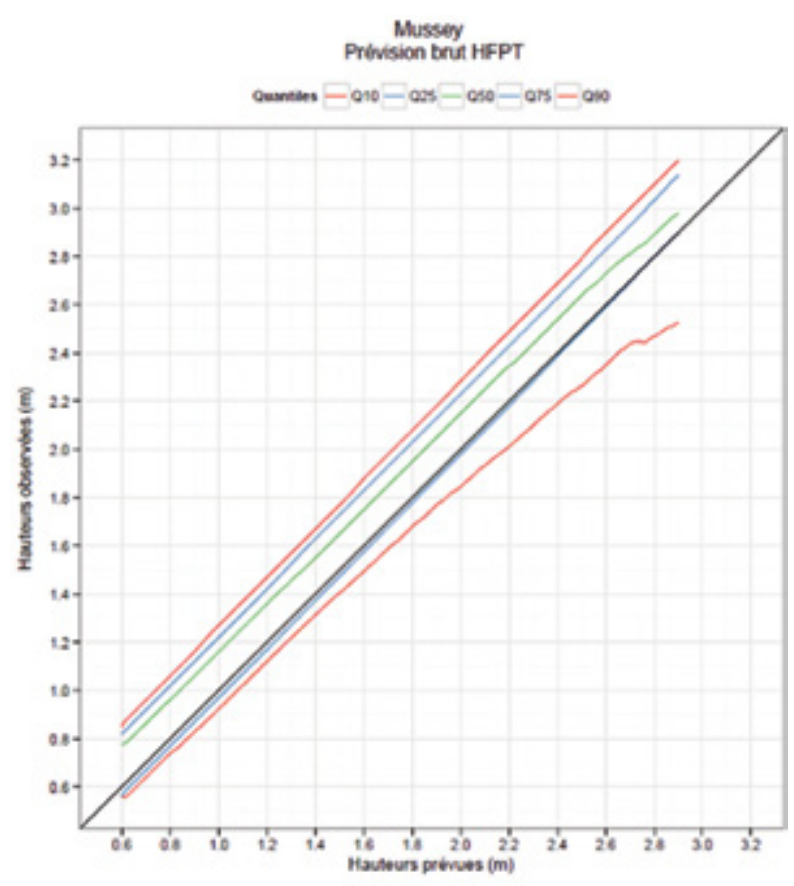

Figure 2 : Exemple de description automatique succincte de l'incertitude sur les prévisions: quantiles $q_{0,10}, q_{0,25}, q_{0,50}$, $q_{0,75}$ et $q_{0,90}$ de la distribution de probabilité de la de hauteur à la station de Mussey, conditionnellement à la hauteur prévue par le modèle MASCARET pour un horizon de $12 \mathrm{~h}$ (en abscisses), obtenus par régression des quantiles. versant, chenal....) et du comportement du modèle. En pratique, le prévisionniste peut sélectionner tout ou partie d'un hydrogramme ou limnigramme (courbe dans un graphe) et le déformer «à la souris ». Ce faisant, il accélère ou ralentit une montée de crue, augmente la valeur d'une pointe... Il exprime ainsi son expertise personnelle et enrichit la prévision au lieu de se contenter de valider les sorties d'un modèle.

\section{III.3. Accompagner les prévisionnistes dans cette évolution}

\section{III.3.1. Formuler un nouveau cadre opérationnel}

Afficher son incertitude n'est pas chose facile pour de nombreuses personnes, a fortiori quand elles sont « sous pression » et placées en position de «sachant» (voire qualifiées, comme souvent un peu trop facilement, « d'experts »). Comme montré par le CST, les attentes des usagers s'expriment principalement dans le cadre d'une culture de la précision. De nombreux travaux dont ceux de Houdant [2004] mettent en évidence la distance qui sépare cette culture technique dominante d'une culture valorisant une information enrichie par le degré de confiance. Aussi, il est nécessaire de préciser le cadre opérationnel du prévisionniste. La formalisation de la mission confiée au prévisionniste (ou des objectifs qui lui sont assignés) doit affirmer qu'il n'est pas attendu qu'il ait « réponse précise à tout " mais qu'il a à énoncer clairement son degré de confiance. Ceci contribue à un «climat de confiance » et répond aux craintes exprimées par certains prévisionnistes d'une remise en cause de leur travail en cas d'affichage d'une "imprécision ». En complément, la culture opérationnelle du service doit rappeler que la raison d'être du prévisionniste (sa plus-value réelle) réside essentiellement dans son expertise, qui doit donc être valorisée dans la production du service.

\section{III.3.2. Les premiers pas}

Au-delà des formations aux concepts liés à l'incertitude et aux outils permettant de la gérer, des premiers vecteurs de sensibilisation sous une forme ludique sont proposés aux SPC. Reprenant des exercices classiques [Houdant, 2004], ils ont pour but de mettre en évidence le décalage fréquent entre l'estimation subjective par tout individu de son « niveau de certitude » (sur des sujets quelconques) et ses connaissances réelles (défaut de sur-confiance ou, plus rarement, de sous-confiance). Cette prise de conscience « personnelle » du prévisionniste conduit au constat de la nécessité d'un entraînement régulier pour y remédier.

Ce travail est complété par la mise à disposition d'un ensemble de fiches techniques rédigées par des prévisionnistes pour des prévisionnistes [Ardilouze et al., 2014], qui aident à la compréhension, à la prise en main des outils et à l'analyse de leurs résultats. Ce « guide» dont le but est de renforcer une culture commune peut également servir aux entraînements. Il est désormais disponible sur http://wikhydro. developpement-durable.gouv.fr.

\section{III.3.3. L'entraînement}

Ces premiers pas sont suivis par des exercices de mise en situation, cette fois spécifiques au métier de prévisionniste. Cet entraînement reproduit les conditions de travail du prévisionniste avec ses contraintes (temps limité, données d'entrée à critiquer....). Il permet également de prendre 
conscience que cette expression de son expertise peut l'aider dans ses autres missions (par exemple, le choix du niveau de vigilance émis par les prévisionnistes des SPC).

Comme le souligne le groupe de travail du CST du SCHAPI, il s'agit d'un travail de longue haleine, qui doit être répété régulièrement et dans la durée. Cette démarche entre dans la "formation continue » du prévisionniste. Ses fruits arriveront à maturité et seront cueillis dans plusieurs années.

\section{III.3.4. L'évaluation : un outil d'aide à l'entraînement}

Enfin, un système d'archivage des prévisions tant brutes (sorties des modèles) qu'expertisées est proposé aux SPC. Il répond à deux objectifs. Il permet d'abord d'enrichir peu à peu l'outil d'estimation automatique de l'incertitude à associer aux prévisions déterministes. De façon similaire, il permet l'examen des prévisions expertisées passées et de vérifier si les estimations d'incertitude sont statistiquement cohérentes. Il s'agit d'une démarche permettant à chaque prévisionniste ou à chaque équipe de s'auto-évaluer et d'améliorer son estimation subjective de l'incertitude dans la durée. Cette démarche n'est possible que dans un cadre formel clair où cette remise en question est attendue (« normale ») et ne remet pas en cause la perception de qualités du prévisionniste.

\section{III.4. Accompagner les usagers de l'information}

Des publics très différents utilisent les prévisions effectuées au sein des SPC. Parmi eux, on distingue les gestionnaires de crise (services de l'État, élus et services des collectivités locales), les usagers spécialisés (comme certains gestionnaires d'ouvrage), les media et le grand public. Le défi est d'accompagner ces publics divers de façon adaptée à chacun d'eux.

Le premier objectif de l'accompagnement est de proposer une " aide à la lecture » des informations fournies qui permettent une bonne compréhension de l'information transmise. Cela passe par la mise en place d'une légende à plusieurs niveaux de lecture. Un premier niveau proposera une information qualitative (des tendances «basse » et « haute », « optimiste » et « pessimiste »)... à condition que soit clairement indiqué qu'il ne s'agit en aucun cas d'un minimum et d'un maximum indépassables. Un second niveau de lecture fera correspondre ces tendances à des niveaux de pari (" $j$ 'ai une chance sur 10 de voir le niveau dépasser la tendance haute. »). Enfin, pour des usagers plus avertis, la légende doit être complétée en indiquant la correspondance recherchée avec les quantiles $q_{0,1}$ et $q_{0,9}$, ouvrant à une utilisation de l'information quantitative (pour la gestion d'ouvrages ou pour la gestion de crise).

Le SCHAPI et les SPC ont engagé, il y a quelques années, un effort pour homogénéiser la rédaction des bulletins de vigilance en standardisant les termes et qualificatifs décrivant les crues et leurs ampleurs. En s'inspirant de ce travail et d'un travail analogue mené par le GIEC [Mastrandrea et al., 2010], la description littérale de l'incertitude dans les bulletins sera cadrée, de façon à construire une lecture constante dans le temps des bulletins, permettant leur pleine compréhension du grand public et des autres usagers.

Par ailleurs, le SCHAPI et les SPC doivent proposer un accompagnement actif aux gestionnaires de crise (en premier lieu, des services de l'État) qui s'inscrit dans les collaborations habituelles des travaux de préparation à la crise (définition des protocoles d'intervention, exercices et simulations), mais aussi dans les retours d'expérience. Les services de gestion de crise s'appuient sur des règles de gestion simples, robustes et généralement binaires basées sur des informations déterministes (par exemple, " s'il est prévu que la cote à l'échelle dépasse $3 \mathrm{~m} 40$ alors je préviens les maires des 5 communes rattachées à cette échelle de crue »). Une incertitude sur leurs données d'entrée (notamment sur les prévisions) peut être perçue comme étant un facteur de complexité et engendrer une réticence à sa prise en compte. L'objectif de l'accompagnement est donc de montrer la possibilité de créer et d'appliquer des règles de gestion, certaines tout aussi simples, d'autres plus multi-critères, permettant de prendre en compte cette incertitude (par exemple, "s'il y a plus de 1 chance sur 10 que la cote à l'échelle dépasse $3 \mathrm{~m}$ 40, alors je lance l'alerte ») et d'en démontrer l'utilité.

\section{CONCLUSIONS}

L'estimation et la communication des incertitudes associées aux prévisions est un enjeu fort des services de prévision pour améliorer la qualité de l'information transmise au public et aux gestionnaires de crise. Le Conseil scientifique et technique du SCHAPI a montré qu'il s'agissait aussi d'un défi technique et surtout d'un défi humain. Le rôle central de l'expertise du prévisionniste est réaffirmé. Le SCHAPI et les SPC préparent activement cette évolution dans le cadre des recommandations du CST. Au-delà, des questions techniques à résoudre, le travail engagé devra être poursuivi dans la durée : il s'agit en effet d'une évolution profonde du cadre de travail et des habitudes à laquelle les prévisionnistes mais aussi les usagers sont invités. Aussi, cette évolution doit être conduite main dans la main avec les usagers dont la participation et les retours sont essentiels pour en assurer la réussite.

\section{RÉFÉRENCES}

Ardilouze C., Audouy J.-N., Berthet L., Boujard O., Chaleon C., Gautheron A., Habert J., Herman A., Janet B., Jurdy N., Kermarec É., Marchandise A., Marty R., Pansu J., Piotte O., Reinbold D., Saunier M., Silva J.-P., Solignac J. Et VillenAVE P. (2014) - Incertitudes en prévision des crues : fiches techniques, http://wikhydro.developpement-durable.gouv. fr/index.php/Catégorie:Incertitudes.

Berthet L. Et Piotte O. (2014) — International survey for good practices in forecasting uncertainty assessment and communication. $E G U$ 2014. 8579

Bourgin F. (2014) - Comment quantifier l'incertitude prédictive en modélisation hydrologique ? Travail exploratoire sur un grand échantillon de bassins versants. Thèse de Doctorat, Agro ParisTech (Paris), Irstea (Antony). 230 p., http://webgr.irstea.fr/ wp-content/uploads/2014/09/2014_BOURGIN_these.pdf

HoudAnT B. (2004) — Contribution à l'amélioration de la prévision hydrométéorologique opérationnelle. Pour l'usage des probabilités dans la communication entre acteurs. Thèse de doctorat de l'ENGREF. Paris. 209 p.

Mastrandrea M.D., C.B. Field, T.F. Stocker, O. Edenhofer, K.L. Ebi, D.J. Frame, H. Held, E. Kriegler, K.J. Mach, P.R. Matschoss, G.-K. Plattner, G.W. Yohe And F.W. ZwIERs (2010) - Guidance Note for Lead Authors of the IPCC Fifth Assessment Report on Consistent Treatment of Uncertainties. Intergovernmental Panel on Climate Change (IPCC). http://www.ipcc.ch 
Morss R.E., J. L. Demuth Et J. K. Lazo (2008) Communicating Uncertainty in Weather Forecasts: A Survey of the U.S. Public. Weather and Forecasting. 23 974-991, DOI 10.1175/2008WAF2007088.1

Nobert S., D. Demeritt Et H. Cloke (2010) — Informing operational flood management with ensemble predictions: lessons from Sweden. Journal of Flood risk management. 3(1) 72-78, DOI 10.1111/j.1753-318X.2009.01056.x

SCHAPI (2013) - Les incertitudes associées aux prévisions des crues et des inondations, leur estimation et leur prise en compte dans la communication. Rapport $d u$ Conseil scientifique et technique.

SCHAPI - SPC (2014) — Rapport du chantier d'intérêt commun sur l'estimation des incertitudes de prévision.

Weerts A. H., Winsemius H. C. And Verkade J. S. (2011) Estimation of predictive hydrological uncertainty using quantile regression: examples from the National Flood Forecasting System (England and Wales). Hydrology and Earth System Sciences. 15 255-265, DOI 10.5194/hess-15-255-2011, 2011 